\title{
EM Isolation Enhancement Based on Metamaterial Concept in Antenna Array System to Support Full-Duplex Application
}

\author{
Mohammad Alibakhshikenari ${ }^{1}$, Marco. Vittori, Sergio. Colangleli, Bal S. Virdee ${ }^{2}$, Aurora Andújar ${ }^{3}$, Jaume \\ Anguera $^{3,4}$, and Ernesto Limiti ${ }^{1}$
${ }^{1}$ Electronics Engineering Department, University of Rome "Tor Vergata", Via del Politecnico 1, 00133 Rome-ITALY
${ }^{2}$ London Metropolitan University, Center for Communications Technology, School of Computing \& Digital Medai, London, N7 8DB, UK
${ }^{3}$ Technology Department, Fractus, 08174 Barcelona, Spain
${ }^{4}$ Department of Electronics and Telecommunication, Universitat Ramon Llull, Barcelona08022, Spain
alibakhshikenari@ing.uniroma2.it, b.virdee@londonmet.ac.uk, aurora.andujar@fractus.com,jaumean@salleurl.edu, limiti@ing.uniroma2.it

\begin{abstract}
This paper proposes EM mechanism to improve the isolation between transmitting and receiving array antennas using metamaterial EM band gap (MTM-EBG). The proposed mechanism can be applied to full-duplex array antenna system with very closely spaced arrays $\left(0.33 \lambda_{0}\right)$ with no degradation in radiation pattern. Using the proposed technique the isolation is shown to improve by $>30 \mathrm{~dB}$ in an antenna array consisting of three-element microstrip patches designed to operate across 9.7 to 12.3 GHz. Parametric analysis was used to optimise the decoupling arrays performances. The proposed antenna array has physical dimensions of $65 \times 22.5 \times 1.6 \mathrm{~mm}^{3}$ and an electrical size of $2.16 \lambda_{0} \times 0.75 \lambda_{0} \times 0.053 \lambda_{0}$, where $\lambda_{0}$ is free space-wavelength at mid-band of $10 \mathrm{GHz}$.
\end{abstract}

Keywords- Mutual coupling suppression, metamaterial, electromagnetic band gap, decoupling arrays, isolation enhancement, full-duplex wireless transceivers.

\section{INTRODUCTION}

In full-duplex applications mutual coupling between the nearby transmitting and receiving antennas results in correlation between the transmitted/received signals, which can significantly degrade the radiation and efficiency characteristics of the antenna [1]. This is because of excitation of surface wave modes [2] that result in mutual coupling between adjacent E-plane coupled microstrip antennas especially when the gap between the antennas is greater than $0.3 \lambda_{0}$ [3]. Numerous techniques have been proposed to date to reduce the mutual coupling between adjacent nearby antennas including (i) using shorting patches to prevent the excitations of the surface waves [4], (ii) using cavity backed slots [5], and (iii) using parasitic elements isolators [6]. Also, metamaterial insulators for small size antennas have been suggested using negative effective permittivity and permeability [7],[8], or reversal of current through the metamaterial radiators [9]. In [10] a parasitic isolator, which is printed between the two patches, is used to suppress mutual coupling. The parasitic isolator controls the polarization of the coupling field to reduce the antenna coupling. Furthermore, a defected ground structure is employed to suppress the cross-polarization level. The measured results show that, at the resonant frequency, the achieved isolation enhancement is $19.6 \mathrm{~dB}$.

In this paper the effectiveness of using metamaterial EM band gap technique to suppress mutual coupling between array elements is demonstrated. Isolation between three elements of a microstrip antenna array is improved by $>30 \mathrm{~dB}$. This is based on combining multiple MTM-EBG decoupling slabs in the array antenna. In addition, by applying this technique size reduction is achieved as the gap between neighbouring antennas can be reduced by $\sim 15 \%$. The three-element antenna array, which was designed to operate across 9.7 to $12.3 \mathrm{GHz}$, has dimensions of $65 \times 22.5 \times 1.6 \mathrm{~mm}^{3}$, and can be applied in the implementation of full-duplex radar.

\section{ISOLATION ENHANCEMENT BETWEEN ANTENNA ARRAY ELEMENTS}

Geometry of the three-element antenna array without MTM-EBG decoupling slab is shown in Fig. 1. The structure was constructed on FR-4 substrate with dielectric constant of $\varepsilon_{r}=4.3$, thickness of $h=1.6 \mathrm{~mm}$ and $\tan \delta=0.025$. The antenna array is composed of three identical circular patches with radius of $15 \mathrm{~mm}$. The proposed antenna array was designed to operate from $9.7 \mathrm{GHz}$ to $12.3 \mathrm{GHz}$, which corresponds to a fractional bandwidth of $23.63 \%$. The average isolation between the radiating elements over its operating range is $-17 \mathrm{~dB}$.

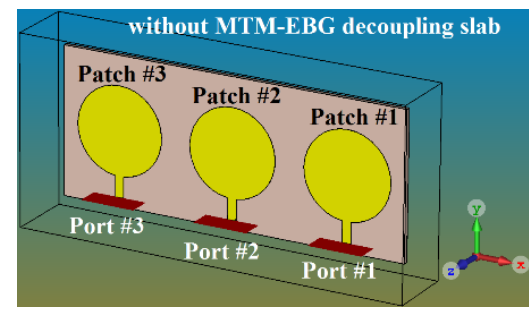


Fig.1. Antenna array constructed with three identical circular patches without MTM-EBG decoupling slab.

To improve the isolation between antenna array elements MTM-EBG decoupling slab is employed. The MTM-EBG is a 'cross' shaped conductor with a cross shaped slot located at its center, as shown in Fig.2. The MTM-EBG decoupling slab is inserted between the circular patches, as depicted in Fig.3.

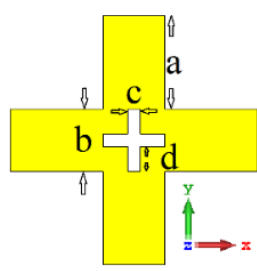

(a)

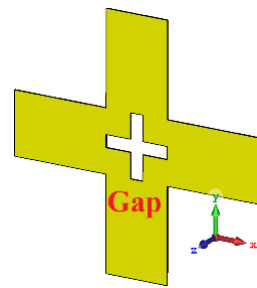

(b)
Fig.2. Single symmetrical metamaterial electromagnetic band gap (MTM-EBG) decupling slab, a) top view, and b) isometric view.

The average EM isolation between the antenna array elements with MTM-EBG decoupling slab is $-27 \mathrm{~dB}$ over its operating range. By inserting MTM-EGB in the antenna array the isolation between the neighbouring radiating elements improved by $59 \%$.

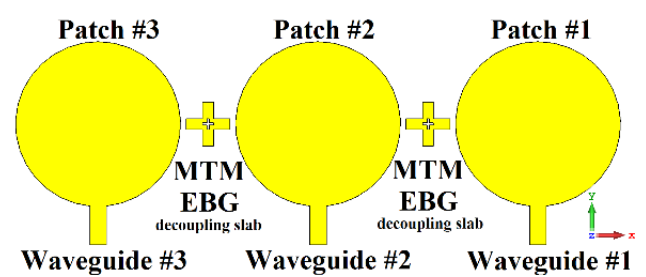

Fig.3. Antenna array with MTM-EBG decoupling slabs located between the radiating elements.

The effect of increasing the number of MTMEBG was also investigated. Fig.4 shows MTM-EBG was increased to three. The dimensions of the antenna array and MTM-EBG decoupling slab are given in Table I. Fig.5 shows how the S-parameter performance of the antenna array is effect by the number of MTMEBG. In all cases the antenna array operates over a frequency range from 9.7 to $12.3 \mathrm{GHz}$. In addition, the antenna match is optimum at $f_{r_{1}}=10.0 \mathrm{GHz}$ and $f_{r_{1}}=11.6 \mathrm{GHz}$ with reflection coefficient of $-25 \mathrm{~dB}$ and $-37 \mathrm{~dB}$, respectively. Average mutual coupling reduction is $-17 \mathrm{~dB}$ with three MTM-EBG, which is an improvement by $20 \mathrm{~dB}$ with a single MTM-EBG. The minimum and maximum isolation between radiation patches in this case are $-25 \mathrm{~dB}$ and $-45 \mathrm{~dB}$, respectively.

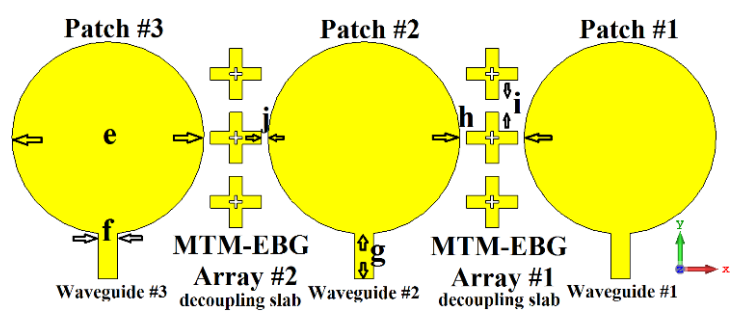

(a) Top view

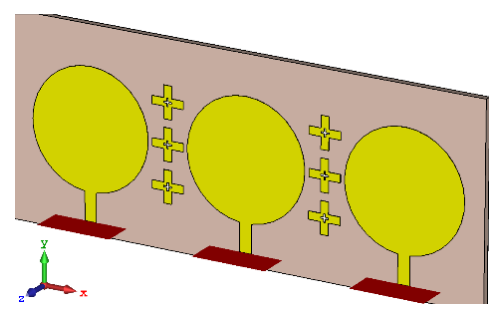

(b) Isometric View

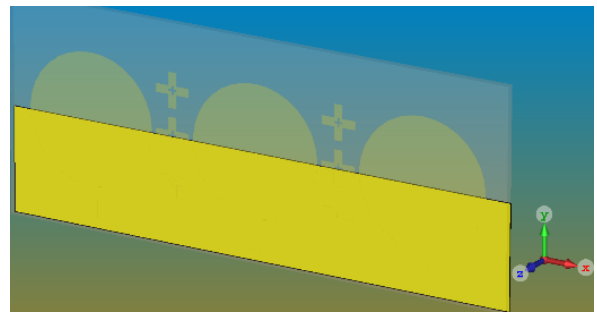

(c) Antenna array's ground plane

Fig.4. Proposed antenna array constructed using three identical circular radiating patches with MTM-EBG decoupling slabs.

TABLE I. DIMENSIONS OF THE PROPOSED ANTENNA ARRAY (Dimension are in millimetres).
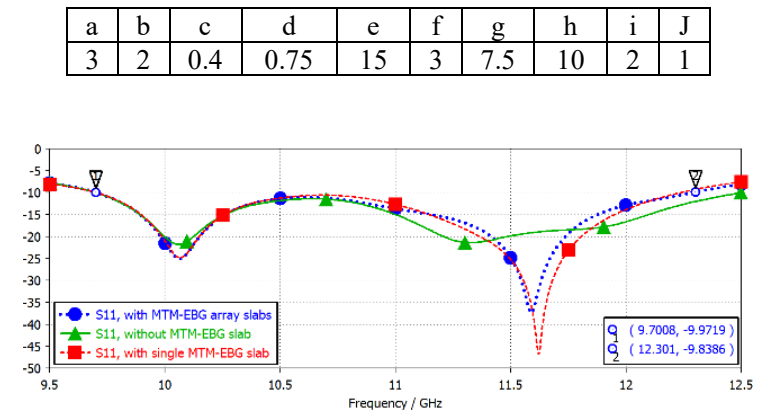

(a) $\mathrm{S}_{11}$

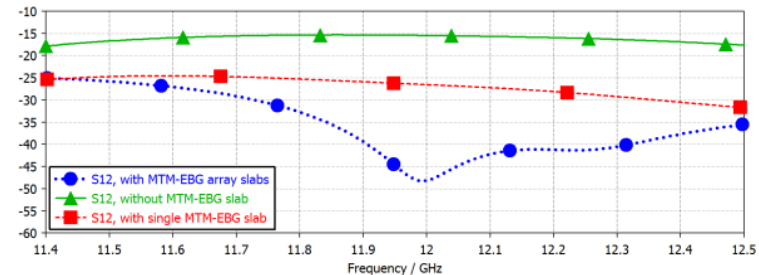

(b) $\mathrm{S}_{12}$

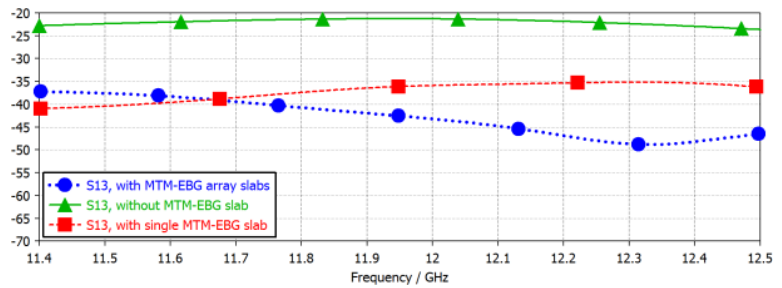

(c) $\mathrm{S}_{13}$ 


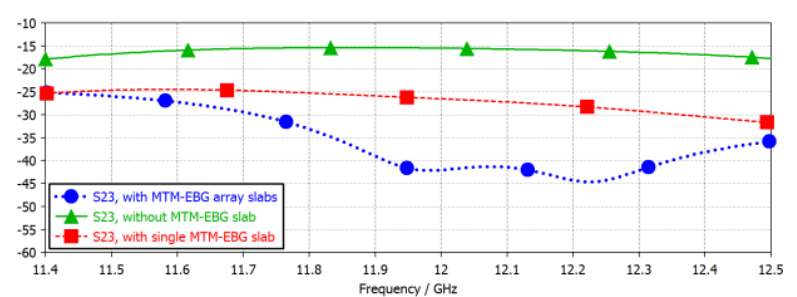

(d) $\mathrm{S}_{23}$

Fig.5. S-parameters performance of the antenna array with three MTM-EBG.

The overall dimensions of the antenna array with MTM-EBG decoupling slab is $65 \times 22.5 \times 1.6 \mathrm{~mm}^{3}$ corresponding to the electrical size of $2.16 \lambda_{0} \times 0.75 \lambda_{0} \times 0.053 \lambda_{0}$, where $\lambda_{0}$ is free spacewavelength at mid-band of $10 \mathrm{GHz}$.

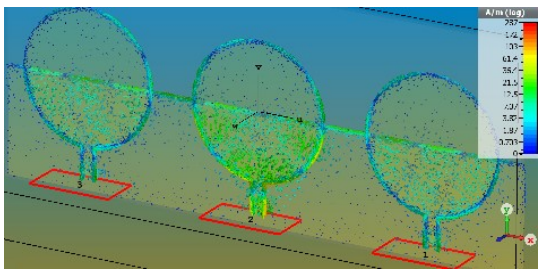

(a)

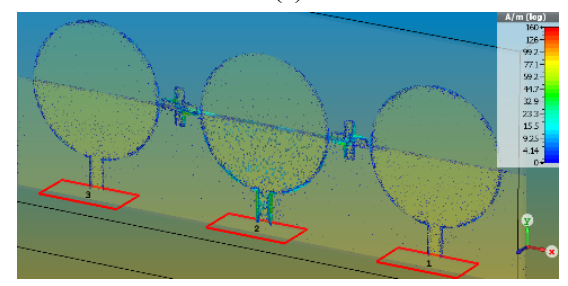

(b)

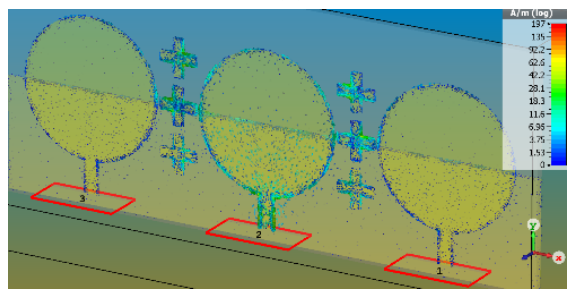

(c)

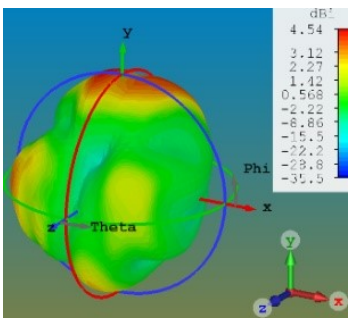

(d)

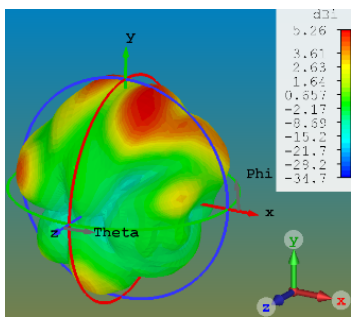

(f)

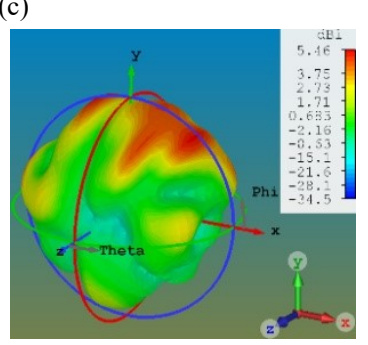

(e)
Fig.6. Antenna array's distributed surface currents and $3-\mathrm{D}$ radiation patterns at resonance frequency of 10 $\mathrm{GHz}$, a) distributed surface current without MTM-EBG decoupling slab, b) distributed surface current with single MTM-EBG decoupling slab, c) distributed surface current with array of MTM-EBG

decoupling slabs, d) 3-D radiation pattern without MTM-EBG decoupling slab, e) 3-D radiation pattern with single MTM-EBG decoupling slab, and f) 3-D radiation pattern with array of MTMEBG decoupling slabs.

For greater insight, the simulated E-field magnitude profile before and after applying the MTMEBG decoupling structure are shown in Figs. 6 (a-c). This shows the propagating E-field is not allowed to be coupled to the nearby antennas, which confirms the effectiveness of the proposed technique in mitigating surface waves. The antenna radiation pattern is enhanced by applying this technique as is evident in Figs. $6(\mathrm{~d}-\mathrm{f})$. 3-D radiation pattern shows greater directivity and efficient behaviour after applying the proposed MTM-EBG decoupling structure. In particular, the maximum gain increased from $2.7 \mathrm{dBi}$ to $6.5 \mathrm{dBi}$. The antenna array was analysed using full wave Microwave CST studio.

\section{CONCLUSION}

Isolation between nearby antenna can be substantially improved $(>3 \mathrm{~dB})$ by simply incorporating an arrangement of metamaterial EM band gap (MTMEBG) decoupling slab between the radiating elements. This is because the proposed MTM-EBG can effectively suppress surface wave modes created on the microstrip antenna. The proposed technique can be applied in the implementation of full-duplex radar as well as in phased array antennas.

\section{REFERENCES}

[1] Pozar, D.M., Schaubert, D.H.: 'Analysis of an infinite array of rectangular microstrip patches with idealized probes feeds', IEEE Trans. Antennas Propag., 1984, 32, (10), pp. 1101-1107.

[2] Pozar, D., Schaubert, D.: 'Microstrip antennas: the analysis and design of microstrip antennas and arrays' (John Wiley \& Sons, 1995).

[3] Jedlicka, R.P., Poe, M.T., Carver, K.R.: 'Measured mutual coupling between microstrip antennas', IEEE Trans. Antennas Propag., 1981, 29, pp. 147-149.

[4] Amendola, G., Boccia, L., Massa, G.: 'Shorted elliptical patch antennas with reduced surface waves on two frequency bands', IEEE Trans. Antennas Propag., 2005, 53, (6), pp. 1946-1956.

[5] Hikage, T., Omiya, M., Itoh, K.: 'Performance evaluation of cavity-backed slot antennas using the FDTD technique'. Proc. of IEEE Antennas and Propagation Society Int. Symp., 2000, vol. 3, pp. 1484-1487.

[6] Zhang, S., Khan, S., He, S.: 'Reducing mutual coupling for an extremely closely-packed tunable dual-element PIFA array through a resonant slot antenna formed in-between', IEEE Trans. Antennas Propag., 2010, 58, (8), pp. 2771-2776.

[7] Buell, K., Mosallaei, H., Sarabandi, K.: 'Metamaterial insulator enabled superdirective array', IEEE Trans. Antennas Propag., 2007, 55, (4), pp. 1074-1085.

[8] Trindade, D.V.B.M., Muller, C., De Castro, M.C.F., et al.: 'Metamaterials applied to ESPAR antenna for mutual coupling reduction', IEEE Antennas Wirel. Propag. Lett., 2014, 14, pp. $1622-1625$.

[9] Abdalla, M., Ibrahim, A.: 'Design of close, compact, and high isolation metamaterial MIMO antennas'. 2013 IEEE AP-S Int. Antenna and Propagation Symp. Digest, Orlando, USA, 7-13 July 2013, pp. 186-187. 
[10] Y.-F. Cheng, X. Ding, W. Shao, B.-Z. Wang, "Reduction of Mutual Coupling Between Patch Antennas Using a PolarizationConversion Isolator, IEEE Antennas and Wireless Propagation Letters, Vol. 16, 2017, pp. 1257-1260. 